\title{
CS-Annotate: A Tool for Using NMR Chemical Shifts to Annotate RNA Structure
}

\author{
Kexin Zhang, ${ }^{\dagger}$ Kyrillos Abdallah,,${ }^{\ddagger}$ Pujan Ajmera, ${ }^{\ddagger}$ Kyle Finos,,${ }^{\ddagger}$ Andrew \\ Looka, ${ }^{\ddagger}$ Joseph Mekhael, ${ }^{\ddagger}$ and Aaron T. Frank ${ }^{*, \ddagger}$ \\ $\dagger$ Chemistry Department, University of Michigan, 930 North University Avenue, Ann \\ Arbor, Michigan 48109, USA \\ $\ddagger$ Biophysics Program, University of Michigan, 930 North University Avenue, Ann Arbor, \\ Michigan 48109, USA \\ E-mail: afrankz@umich.edu \\ Phone: (734) 615-2053
}




\section{Feature Distribution}

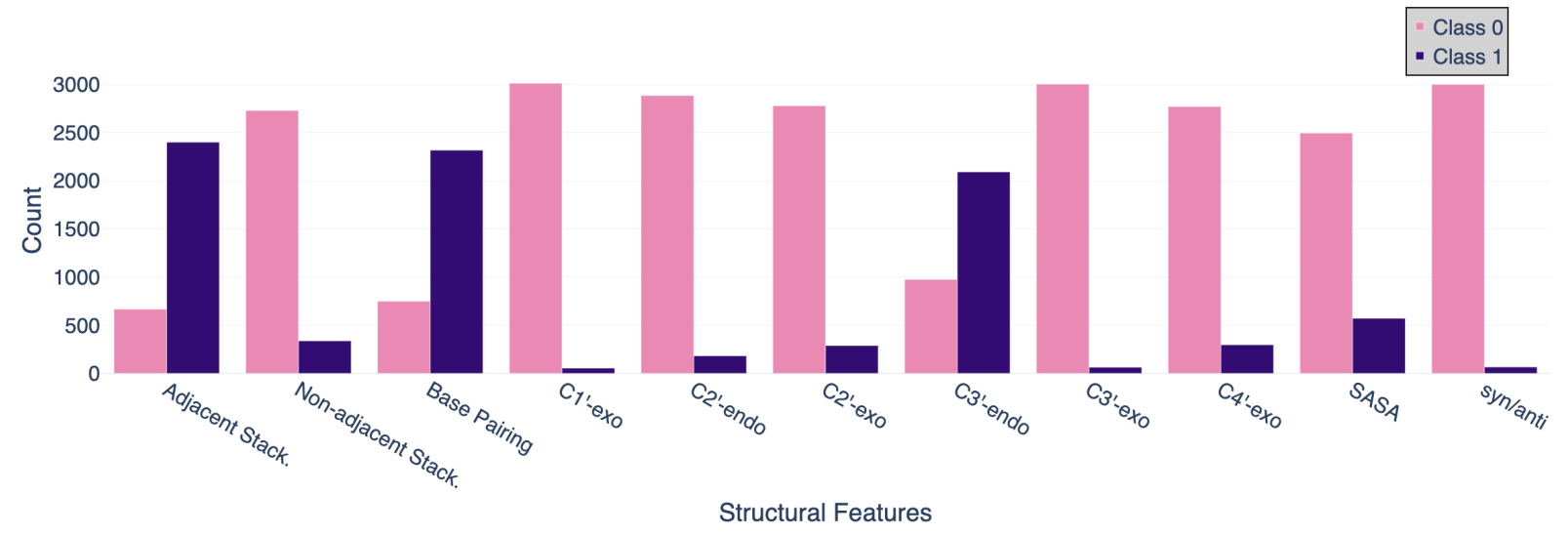

Figure S1: Distribution of annotation targets (structural properties) in the training set. Class 1 is the positive class (property present) and Class 0 is the negative class (property absent). "Adjacent Stack." represents stacking interaction between adjacent bases while "Non-adjacent Stack." represents non-adjacent stacking interaction. 


\section{Comparison to baseline models}

Table S1: Comparison between the multi-task progressive neural network (PNN) model used by CS-Annotate and seven independent baseline models.

\begin{tabular}{|c|c|c|c|c|c|c|c|c|c|}
\hline Property & PNN & Model $^{1}$ & Model $^{2}$ & Model $^{3}$ & Model $^{4}$ & Model $^{5}$ & Model $^{6}$ & Model $^{7}$ & Mod \\
\hline djace & 699 & 690 & 0.674 & 0.638 & 674 & 0.681 & 0.646 & .649 & 499 \\
\hline TNOn & 8 & & 4 & & & 27 & & & 500 \\
\hline Bas & 312 & 69 & 5 & 5 & 6 & 3 & 5 & 5 & 00 \\
\hline C1'- & 342 & 0.521 & 783 & 81 & 07 & +1 & 14 & 99 & 500 \\
\hline C2'- & 787 & 0.634 & 0.677 & 0.668 & 0.605 & 0.627 & 0.511 & 587 & .500 \\
\hline C2' & 8 & 0.5 & 0.6 & 0. & & 0. & 9 & 2 & 00 \\
\hline C & 1 & 0.6 & 0. & 0. & 0. & & & & 86 \\
\hline C3' & 780 & 0.5 & 0. & & & & & & 98 \\
\hline C & 5 & 0.5 & & 0. & & & & & 00 \\
\hline SA & & & & & & & & & 0.500 \\
\hline syn/anti & 0.762 & 0.684 & 0.530 & 0.720 & 0.668 & 0.678 & 0.556 & 0.662 & 0.500 \\
\hline mean & 708 & 0.631 & 0.632 & 0.632 & 0.619 & 0.635 & 0.574 & 0.607 & .498 \\
\hline
\end{tabular}

${ }^{1}$ Independent multilayer perceptron (MLP) classifiers. MLP classifiers each trained to predict one of the 11 structural properties from chemical shifts. The model is a single layer MLP classifier with 100 hidden neurons. The activation function used was a rectified linear unit (ReLU).

2 Recurrent neural network classifier. This model is a 2 layer Long Short-Term Memory (LSTM) RNN with 128 hidden units and a dropout of 0.3 . The RNN reads the data sequentially on the "residue"axis and leverage a multi-task classifier consisting of a fully connected 512-unit layer at each residue. LeakyReLU activation and Sigmoid activation were used before and after the fully connected layer, respectively. Pack_padded_sequence and masking the loss function (Binary Cross Entropy Loss) was used to handle variable length inputs.

3 1D convolutional classifier. This model is a 1-dimensional, single-task, graph convolution model. The model used a single 1D convolutional deep layer (6 kernels, each with kernel size 3 ) to detect for patterns across the chemical shifts. The activation was sigmoid for the input and output layers, compiled with a binary loss entropy loss function and adam optimizer.

${ }^{4}$ Random Forest/multi-task MLP Ensemble Classifier. This model used random forest classifiers in conjunction with a multitask neural network classifier. The random forest classifiers used a balanced weight class and consisted of 100 trees with a maximum depth of 10 . The probability values from the random forest classifiers were saved and incorporated into the dataset for the multitask classifier. The multitask classifier consisted of 4 layers of 1000 neurons with a dropout of 0.5 and a ReLU activation function.

${ }^{5}$ Chained Ensemble Classifier. In the chained classifiers, predictions of the previous properties were used as input for the following predictions. The chained ensemble classifier combined results from chains with a different order of properties. Individual classifiers within the chained ensemble classifier had the same architecture as the independent MLP classifier.

${ }^{6}$ Random Forest Classifier. Classifier was trained using sklearn (version 0.23) and contains 100 trees. The Gini impurity was used as the training criterion. Default values were used for all other training parameters.

7 Logistic Regression Classifier. Classifier was trained using sklearn (version 0.23) and using L2 penalty function. Default values were used for all other training parameters.

8 Y-scrambled Classifier. Same as the PNN model, expect that the target data was scrambled prior to training the classifier. 


\section{Sensitivity and specificity of testing set}

Table S2: Sensitivity and specificity of testing set. ${ }^{1}$

\begin{tabular}{lll}
\hline Property & Sensitivity & Specificity \\
\hline Solvent Exposure & 0.923 & 0.778 \\
Adjacent stacking & 0.780 & 0.533 \\
Non-adjacent stacking & 0.471 & 0.912 \\
Base-pairing & 0.845 & 0.758 \\
Syn or Anti & 0.667 & 0.961 \\
C2'-endo & 1.000 & 0.942 \\
C3'-endo & 0.847 & 0.511 \\
C2'-exo & 0.308 & 0.740 \\
C3'-exo & 0.333 & 0.921 \\
C1'-exo & 1.000 & 0.906 \\
C4'-exo & 0.200 & 0.816 \\
\hline Mean & 0.670 & 0.798 \\
\hline
\end{tabular}

${ }^{1}$ sensitivity and specificity values from an independent testing set not included in the training set. The testing set consists of data for the Simian immunodeficiency virus (SIV) frameshift-inducing RNA (PDB ID: 2JTP), the $\kappa-\zeta$ region of S.cerevisiae group II intron ai5 $(\gamma)$ RNA (PDB ID: 2LU0), and the fluoride riboswitch (PDB ID: $5 \mathrm{KH} 8$ ) 


\section{Receiver-operator characteristic (ROC) analysis}

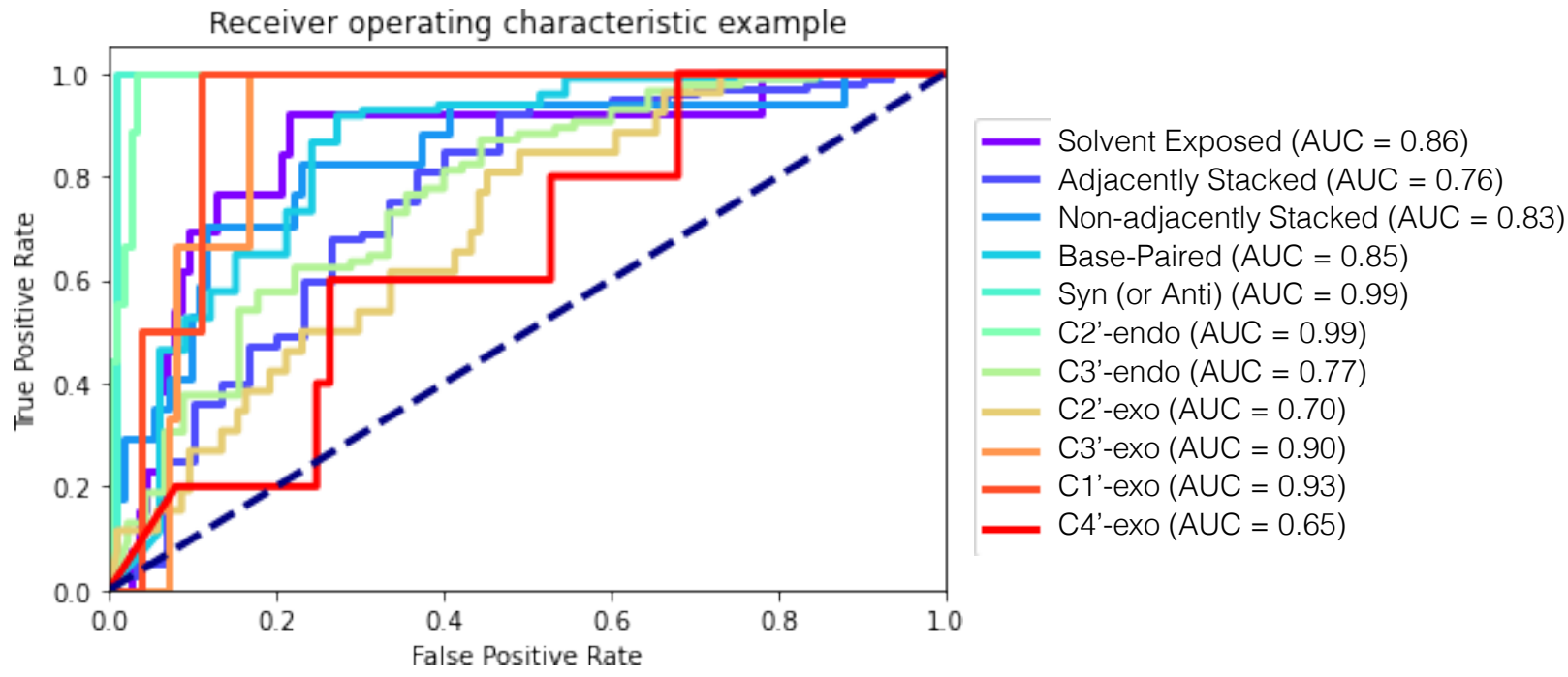

Figure S2: ROC curves and AUCs for the 11 targets across the testing set. Here ROC were calculated using the classification probabilities from our multi-task classifier. 


\section{Impact of neighboring residues}

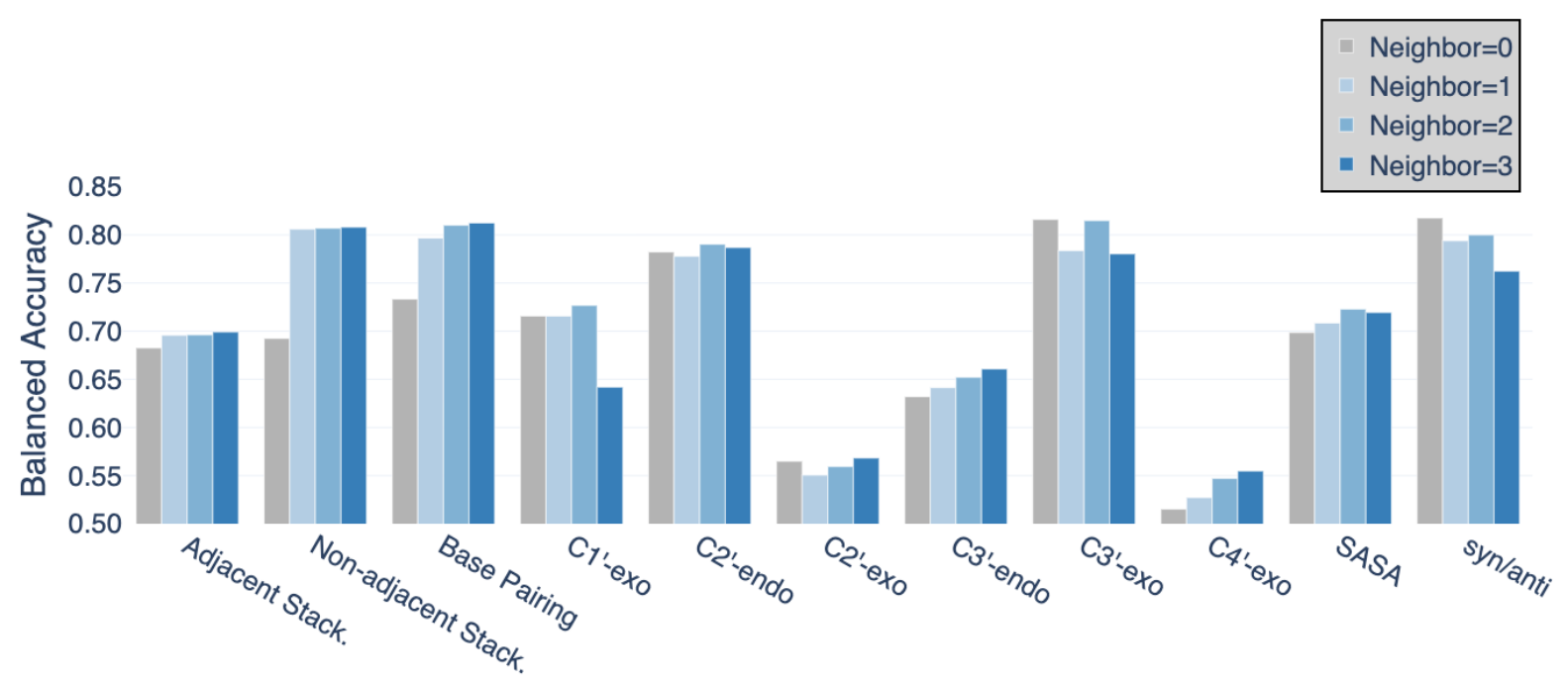

Figure S3: Balanced accuracy of validation sets when including different number of neighboring residues. In most cases, the accuracy increases with the number of neighboring chemical shift and residues used to describe a given residue $i$. When consider $m$ neighboring residues as features: for residue $i$, the features are the chemical shifts and nucleotide types for residues $i-m, . ., i, . ., i+m$. In most cases, the accuracy increase with the $m$. 